\title{
AN EFFICIENT REFINEMENT STRATEGY EXPLOITING COMPONENT PROPERTIES IN A CEGAR PROCESS
}

\author{
Syed Hussein S. ALWI, Cécile BRAUNSTEIN and Emmanuelle ENCRENAZ \\ Université Pierre et Marie Curie Paris 6, \\ LIP6-SOC (CNRS UMR 7606), \\ 4, place Jussieu, \\ 75005 Paris, FRANCE.
}

\begin{abstract}
Embedded systems are usually composed of several components and in practice, these components generally have been independently verified to ensure that they respect their specifications before being integrated into a larger system. Therefore, we would like to exploit the specification (i.e. verified CTL properties) of the components in the objective of verifying a global property of the system. A complete concrete system may not be directly verifiable due to the state explosion problem, thus abstraction and eventually refinement process are required. In this paper, we propose a technique to select properties in order to generate a good abstraction and reduce refinement iterations. We have conducted several preliminary experimentations which show that our approach is promising in comparison to other abstraction-refinement techniques implemented in VIS [1].
\end{abstract}

Index Terms - Compositional verification, CTL properties, CEGAR, model-checking

\section{INTRODUCTION}

The embedded systems correspond to the integration into the same electronic circuit, a huge number of complex functionalities performed by several heterogeneous components. Current SoC (System on Chips) contain multiple processors executing numerous cooperating tasks, specialized co-processors (for particular data treatment or communication purposes), Radio-Frequency components, etc. These systems are usually submitted to safety and robustness requirements. Depending on their application domains, their failure may induce serious damages and catastrophic consequences.

Therefore, it is important to ensure, during their design phase, their correctness with respect to their specifications. Errors found late in the design of these systems is a major problem for electronic circuit designers and programmers as it may delay getting a new product to the market or cause failure of some critical devices that are already in use. System verification using formal methods such as model checking guarantees a high level of quality in terms of safety and reliability while reducing financial risk.

The main challenge in model checking is dealing with the state space combinatorial explosion phenomenon. A strategy to overcome the state explosion problem is by performing abstraction. A method for the construction of an abstract state graph of an arbitrary system automatically was first proposed by Graf and Saidi [2] using Pvs theorem prover. Here, the abstract states are generated from the valuations of a set of predicates on the concrete variables. The construction approach is automatic and incremental.

In 2000, an interesting abstraction-refinement methodology called counterexample-guided abstraction refinement (CEGAR) was proposed by Clarke and al. [3]. The abstraction was done by generating an abstract model of the system by considering only the variables that possibly have a role in verifying a particular property. In this technique, the counterexample provided by the model-checker in case of failure is used to refine the system.

Several tools using counterexample-guided abstraction refinement technique, like those implemented in the VIS model-checker, have been developed such as SLAM, a software model-checker by Microsoft Research [4], BLAST (Berkeley Lazy Abstraction Software Verification Tool), a software model-checker for C programs [5] and VCEGAR (Verilog Counterexample Guided Abstraction Refinement), a hardware model-checker which performs verification at the RTL (Register Transfer Language) level [6]. However, relying on counterexamples generated by the model checker as the only source for refinement may not be conclusive.

Recently, a CEGAR based technique that combines precise and approximated methods within one abstractionrefinement loop was proposed for software verification [7]. This technique uses predicate abstraction and provides a strategy that interleaves approximated abstraction which 
is fast to compute and precise abstraction which is slow. The result shows a good compromise between the number of refinement iterations and verification time.

An alternative method to get over the state explosion problem is the compositional strategy. The strategy is based on the assume-guarantee reasoning where assumptions are made on other components of the systems when verifying one component. Several works have manipulated this technique notably in [8] where Grumberg and Long described the methodology using a subset of CTL in their framework and later in [9] where Henzinger and al. presented their successful implementations and case study regarding this approach.

Xie and Browne have proposed a method for software verification based on composition of several components [10]. Their main objective is developing components that could be reused with certitude that their behaviors will always respect their specification when associated in a proper composition. Therefore, temporal properties of the software are specified, verified and packaged with the component for possible reuse. The implementation of this approach on software has been successful and the application of the assume-guarantee reasoning has considerably reduced the model checking complexity. A comprehensive approach to model-check component-based systems with abstraction refinement technique that uses verified properties as abstractions has been presented in [11].

In [12], Peng, Mokhtari and Tahar have presented a possible implementation of assume-guarantee approach where the specifications are in ACTL. Moreover, they managed to perform the synthetisation of the ACTL formulas into Verilog HDL behavior level program. The synthesized program can be used to check properties that the system's components must guarantee. Since, there have been other works on construction of components from interval temporal logic properties which could be used to speed up verification process [13] [14].

In 2007, a method to build abstractions of components into AKS (Abstract Kripke Structure), based on the set of the properties (CTL) each component verifies was presented in [15]. The method is actually a tentative to associate compositional and abstraction-refinement verification techniques. The generations of AKS from CTL formula have been successfully automated [16]. This work will be the base of the techniques in this paper.

Contribution : In this paper we present a strategy to exploit the properties of verified component in the goal of verifying complex systems with a good initial abstraction and eventually being conclusive in minimal refinement iterations. We propose a technique to classify component properties according to their pertinency towards the global property, thus, enabling a better selection of properties for the initial abstraction generation. Furthermore, in the case where the verification is not con- clusive, we propose a technique guided by the counterexample given by the model-checker to select supplementary properties to improve the abstraction.

In the next section, we will give an overview of our framework and introduce the notations that will be used later. The rest of the paper is organized as follows: section 3 details our strategy of refinement. Section 4 presents the experimentation results and finally, section 5 draws the conclusions and summarize our possible future works.

\section{OUR FRAMEWORK}

The model-checking technique we propose is based on the Counterexample-guided Abstraction Refinement (CEGAR) methodology [3]. The overall description of our methodology is shown in Figure 1. We take into account the structure of the system as a set of synchronous components, each of which has been previously verified and a set of CTL properties is attached to each component. This set refers to the specification of the component. We would like to verify whether a concrete model, $M$ presumably big sized and composed of several components, satisfies a global ACTL property $\Phi$. Instead of building the product of the concrete components, we replace each concrete component by an abstraction of its behavior derived from a subset of the CTL properties it satisfies. Each abstract component represents an over-approximation of the set of behaviors of its related concrete component [15].

As shown in [17] for over-approximation abstraction, if $\Phi$ holds in the abstract model then it holds in the concrete model as well. However, if $\Phi$ does not hold in the abstract model then one cannot conclude anything regarding the concrete model until the counterexample has been analyzed. The test of spurious counter-example is then translated into a SAT problem as in [3]. When a counterexample is proven to be spurious, the refinement phase occurs, injecting more preciseness into the (abstract) model to be analyzed.

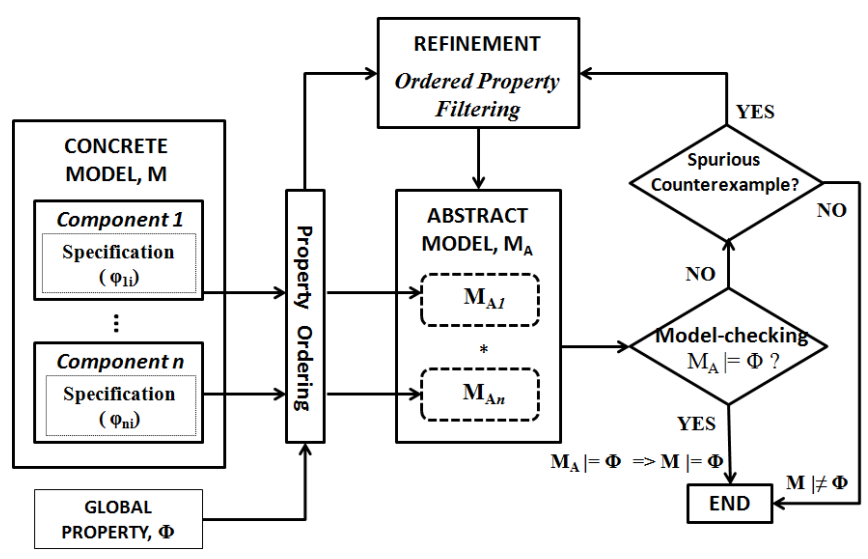

Fig. 1. Verification Process 


\subsection{Concrete system definition}

As mentioned earlier, our concrete model consists of several components and each component comes with its specification. The concrete system is a synchronous composition of components, each of which described as a Moore machine.

Definition 1. A Moore machine $C$ is defined by a tuple $\left\langle I, O, R, \delta, \lambda, \mathbf{R}_{0}\right\rangle$, where,

- I is a finite set of Boolean input signals.

- $O$ is a finite set of Boolean output signals.

- $R$ is a finite set of Boolean sequential elements (registers).

- $\delta: 2^{I} \times 2^{R} \rightarrow 2^{R}$ is the transition function.

- $\lambda: 2^{R} \rightarrow 2^{O}$ is the output function.

- $\mathbf{R}_{0} \subseteq 2^{R}$ is the set of initial states.

States (or configurations) of the circuit correspond to Boolean configurations of all the sequential elements.

Definition 2. A Concrete system $M$ is obtained by synchronous composition of the component.

$M=C_{1}\left\|C_{2}\right\| \ldots \| C_{n}$, where each $C_{i}$ is a Moore machine with a specification associated $\varphi_{i}=\left\{\varphi_{i}^{1} \ldots \varphi_{i}^{k}\right\}$. Each $\varphi_{i}^{j}$ is a $C T L \backslash X$ formula whose propositions $A P$ belong to $\left\{I_{i} \cup O_{i} \cup R_{i}\right\}$.

\subsection{Abstraction definition}

Our abstraction reduces the size of the representation model by letting free some of its variables. The point is to determine the good set of variable to be freed and when to free them. We take advantage of the CTL specification of each component: a CTL property may be seen as a partial view of the tree of behaviors of its variables. All the variables not specified by the property can be freed. We introduced the Abstract Kripke Structure (AKS for short) which exactly specifies when the variable of the property can be freed. The abstraction of a component is represented by an AKS, derived from a subset of the CTL properties the component satisfies. Roughly speaking, $\operatorname{AKS}(\varphi)$, the AKS derived from a CTL property $\varphi$, simulates all execution trees whose initial state satisfies $\varphi$. In $\operatorname{AKS}(\varphi)$, states are tagged with the truth values of $\varphi$ 's atomic propositions, among the four truth values of Belnap's logic [18]: inconsistent $(\perp)$, false (f), true $(\mathbf{t})$ and unknown $(T)$ ). States with inconsistent truth values are not represented since they refer to non possible assignments of the atomic propositions. A set of fairness constraints eliminates non-progress cycles. The transformation algorithm of a $\mathrm{CTL} \backslash \mathrm{X}$ property into an AKS is described in $[15,19]$.

Definition 3. Given a $C T L \backslash X$ property $\varphi$ whose set of atomic propositions is $A P, A n$ Abstract Kripke Structure, $A K S(\varphi)=\left(A P, \widehat{S}, \widehat{S}_{0}, \widehat{L}, \widehat{R}, \widehat{F}\right)$ is a 6 -tuple consisting of:
- AP : The finite set of atomic propositions of property $\varphi$

- $\widehat{S}$ : a finite set of states

- $\widehat{S}_{0} \subseteq \widehat{S}$ : a set of initial states

- $\widehat{L}: \widehat{S} \rightarrow \mathcal{B}^{|\mathcal{A P}|}$ with $\mathcal{B}=\{\perp, \mathbf{f}, \mathbf{t}, \top\}$ : a labeling function which labels each state with configuration of current value of each atomic proposition.

- $\widehat{R} \subseteq \widehat{S} \times \widehat{S}:$ a transition relation where $\forall s \in$ $\widehat{S}, \exists s^{\prime} \in \widehat{S}$ such that $\left(s, s^{\prime}\right) \in \widehat{R}$

- $\widehat{F}$ : a set of fairness constraints (generalized Büchi acceptance condition)

We denote by $\widehat{L}(s)$, the configuration of atomic propositions in state $s$, and by $\widehat{L}(s)[p]$, the projection of configuration $\widehat{L}(s)$ according to atomic proposition $p$.

As the abstract model $\widehat{M}$ is generated from the conjunction of verified properties of the components in the concrete model $M$, it can be seen as the composition of the AKS of each property. The AKS composition has been defined in [19]; it extends the classical synchronous composition of Moore machine to deal with four-valued variables.

Definition 4. An Abstract model $\widehat{M}$ is obtained by synchronous composition of components abstractions. Let $n$ be the number of components in the model and $m$ be the number of selected verified properties of a component; let $C_{j}$ be a component of the concrete model $M$ and $\varphi_{j}^{k}$ is a CTL formula describing a satisfied property of component $C_{j}$. Let $A K S\left(\varphi_{C_{j}^{k}}\right)$ the AKS generated from $\varphi_{j}^{k}$. We have $\forall j \in[1, n]$ and $\forall k \in[1, m]$ :

$$
\begin{aligned}
& \text { - } \widehat{C}_{j}=A K S\left(\varphi_{C_{j}^{1}}\right)\left\|A K S\left(\varphi_{C_{j}^{2}}\right)\right\| \ldots\left\|A K S\left(\varphi_{C_{j}^{k}}\right)\right\| \\
& \quad \ldots \| A K S\left(\varphi_{C_{j}^{m}}\right) \\
& \text { - } \widehat{M}=\widehat{C}_{1}\left\|\widehat{C}_{2}\right\| \ldots\left\|\widehat{C}_{j}\right\| \ldots \| \widehat{C}_{n}
\end{aligned}
$$

In an AKS, a state where a variable $p$ is unknown can simulate all states in which $p$ is either true or false. It is a concise representation of the set of more concrete states in which $p$ is either true or false. A state $s$ is said to be an abstract state if one of its variable $p$ is unknown.

Definition 5. The concretization of an abstract state $s$ with respect to the variable $p$ (unknown in that state), assigns either true or false to $p$.

The abstraction of a state $s$ with respect to the variable $p$ (either true or false in that state), assigns unknown to $p$.

Property 1 (Concretization). Let $A_{i}$ and $A_{j}$ two $a b$ stractions such that $A_{j}$ is obtained by concretizing one abstract variable of $A_{i}$ (resp. $A_{i}$ is obtained by abstracting one variable in $A_{j}$ ). Then $A_{i}$ simulates $A_{j}$, denoted by $A_{j} \sqsubseteq A_{i}$. 
Proof. As the concretization of state reduces the set of concrete configuration the abstract state represents but does not affect the transition relation of the AKS. The unroll execution tree of $A_{j}$ is a sub-tree of the one of $A_{i}$. Then $A_{i}$ simulates $A_{j}$.

Property 2 (Composition and Concretization). Let $\widehat{M}_{i}$ be an abstract model of $M$ and $\varphi_{j}^{k}$ be a property of a component $C_{j}$ of $M, \widehat{M}_{i+1}=\widehat{M}_{i} \| A K S\left(\varphi_{j}^{k}\right)$ is more concrete that $\widehat{M}_{i}, \widehat{M}_{i+1} \sqsubseteq \widehat{M}_{i}$.

Proof. Let $s=\left(s_{i}, s_{\varphi_{j}^{k}}\right)$ be a state in $S_{i+1}$, such that $s_{i} \in S_{i}$ and $s_{\varphi_{j}^{k}} \in S_{\varphi_{j}^{k}}$. The label of $s_{i+1}$ is obtained by applying the Belnap's logic operators and to the fourvalued values of variables in $s_{i}$ and $s_{\varphi_{j}^{k}}$. For all $p \in$ $A P_{i} \cup A P_{\varphi_{j}^{k}}$ we have the following label :

- $\widehat{L}_{i+1}[p]=\top$ iff $\mathrm{p}$ is unknown in both states or does not belong to the set of atomic proposition.

- $\widehat{L}_{i+1}[p]=\mathbf{t}$ (or $\mathbf{f}$ ) iff $p$ is true (or false) in $s_{\varphi_{j}^{k}}$ (resp. $\left.s_{i}\right)$ and unknown in $s_{i}\left(\operatorname{resp} . s_{\varphi_{j}^{k}}\right)$.

By Property 1, $\widehat{M}_{i+1}$ is more concrete than $\widehat{M}_{i}$ and by the property of parallel composition, $\widehat{M}_{i} \sqsubseteq \widehat{M}_{i} \| A K S\left(\varphi_{j}^{k}\right)$.

\subsection{Initial abstraction}

Given a global property $\Phi$, the property to be verified by the composition of the concrete components model, an abstract model is generated by selecting some of the properties of the components which are relevant to $\Phi$. In the initial abstraction generation, all variables that appear in $\Phi$ have to be represented. Therefore the properties in the specification of each component where these variables are present will be used to generate the initial abstraction, $\widehat{M}_{0}$ and we will verify the satisfiability of the global property $\Phi$ on this abstract model. If the modelchecking failed and the counterexample given is found to be spurious, we will then proceed with the refinement process.

\section{REFINEMENT}

\subsection{Properties of good refinement}

When a counterexample is found to be spurious, it means that the current abstract model $\widehat{M}_{i}$ is too coarse and has to be refined. In this section, we will discuss about the refinement technique based on the integration of more verified properties of the concrete model's components in the abstract model to be generated. Moreover, the refinement step from $\widehat{M}_{i}$ to $\widehat{M}_{i+1}$ respects the properties below:

Definition 6. An efficient refinement verifies the following properties:
1. The new refinement is an over-approximation of the concrete model: $\widehat{M} \sqsubseteq \widehat{M}_{i+1}$.

2. The new refinement is more concrete than the previous one: $\widehat{M}_{i+1} \sqsubseteq \widehat{M}_{i}$.

3. The spurious counterexample in $\widehat{M}_{i}$ is removed from $\widehat{M}_{i+1}$.

Furthermore, the refinement steps should be easy to compute and ensure a fast convergence by minimizing the number of iterations of the CEGAR loop.

Refinements based on the concretization of selected abstract variables in $\widehat{M}_{i}$ ensure item 2 . Concretization can be performed by modifying the AKS of $\widehat{M}_{i}$ by changing some abstract value to concrete ones. However, this approach is rude: in order to ensure item 1, the concretization needs to be consistent with the sequences of values in the concrete system. The difficulty resides in defining the proper abstract variable to concretize, at which precise instant, and with which Boolean value.

We propose to compose the abstraction with another AKS to build a good refinement according to Definition 6 . We have several options. The most straightforward method consists in building an AKS representing all possible executions except the spurious counterexample; however the AKS representation may be huge and the process is not guaranteed to converge. A second possibility is to build an AKS with additional CTL properties of the components; the AKS remains small but item 3 is not guaranteed, hence delaying the convergence. The final proposal combines both previous ones: first local CTL properties eliminating the spurious counterexample are determined, and then the corresponding AKS is synchronized with the one of $\widehat{M}_{i}$.

\subsection{Negation of the counterexample}

The counterexample at a refinement step $i, \sigma$, is a path in the abstract model $\widehat{M}_{i}$ which dissatisfies $\Phi$. In the counterexample given by the model-checker, the variable configuration in each state is Boolean. We name $\widehat{L_{i}}$ this new labeling. The spurious counterexample $\sigma$ is defined such that:

Definition 7. Let $\sigma$ be a spurious counterexample in $\widehat{M}_{i}=\left\langle\widehat{A P}_{i}, \widehat{S}_{i}, \widehat{S}_{0 i}, \widehat{L}_{i}, \widehat{R}_{i}, \widehat{F}_{i}\right\rangle$ of length $|\sigma|=n: \sigma=$ $s_{0} \rightarrow s_{1} \ldots \rightarrow s_{n}$ with $\left(s_{k}, s_{k+1}\right) \in \widehat{R}_{i} \forall k \in[0 . . n-1]$.

- All its variables are concrete: $\forall s_{i}$ and $\forall p \in \widehat{A P}_{i}$, $p$ is either true or false according to $\widehat{L_{i}}$. (not unknown), and $s_{0}$ is an initial state of the concrete system: $s_{0} \in \mathbf{R}_{0}$

- $\sigma$ is a counterexample in $\widehat{M}_{i}: s_{0} \not \models \Phi$.

- $\sigma$ is not a path of the concrete system $M: \exists k \in$ $[1 . . n-1]$ such that $\forall j<k,\left(s_{j}, s_{j+1}\right) \in R$ and $\left(s_{k}, s_{k+1}\right) \notin R$. 
The construction of the AKS representing all executions except the one described by the spurious counterexample is done in two steps.

\subsubsection{Step 1 : Build the structure of the AKS.}

Definition 8. Let $\sigma$ be a spurious counterexample of length $|\sigma|=n$, the AKS of the counterexample negation $A K S(\bar{\sigma})=\left\langle\widehat{A P}_{\bar{\sigma}}, \widehat{S}_{\bar{\sigma}}, \widehat{S}_{0 \bar{\sigma}}, \widehat{L}_{\bar{\sigma}}, \widehat{R}_{\bar{\sigma}}, \widehat{F}_{\bar{\sigma}}\right\rangle$ is such that:

- $A P_{\bar{\sigma}}=A P_{i}$ : The set of atomic propositions coincides with the one of $\sigma$

- $\widehat{S}_{\bar{\sigma}}:\left\{s_{T}\right\} \cup\left\{s_{i}^{\prime} \mid \forall i \in[0 . . n-2] \wedge s_{i} \in \sigma\right\} \cup\left\{\bar{s}_{i} \mid \forall i \in\right.$ $\left.[0 . . n-1] \wedge s_{i} \in \sigma\right\}$

- $\widehat{L}_{\bar{\sigma}}$ with $L_{\bar{\sigma}}\left(s_{i}^{\prime}\right)=L_{i}\left(s_{i}\right), \forall i \in[0 . . n-2]$ and $L\left(s_{T}\right)=$ $\left\{\top, \forall p \in A P_{\bar{\sigma}}\right\}, L_{\bar{\sigma}}\left(\bar{s}_{i}\right)$ is explained in the next construction step.

- $\widehat{S}_{0 \bar{\sigma}}=\left\{s_{0}^{\prime}, \overline{s_{0}}\right\}$

- $\widehat{R}_{\bar{\sigma}}=\left\{\left(\bar{s}_{i}, s_{T}\right), \forall i \in[0 . . n-1]\right\} \cup\left\{\left(s_{i}^{\prime}, \overline{s_{i+1}}\right), \forall i \in\right.$ $[0 . . n-2]\} \cup\left\{\left(s_{i}^{\prime}, s_{i+1}^{\prime}, \forall i \in[0 . . n-3]\right\}\right.$

- $\widehat{F}_{\bar{\sigma}}=\emptyset$

The labeling function of $s_{i}^{\prime}$ represents (concrete) configuration of state $s_{i}$ and state $\overline{s_{i}}$ represents all configurations but the one of $s_{i}$. This last set may not be representable by the labeling function defined in Definition 3. State labeling is treated in the second step. $s_{T}$ is a state where all atomic propositions are unknown.

\subsubsection{Step 2 : Expand state configurations representing the negation of a concrete configuration.}

The set of configurations associated with a state $\bar{s}_{i}$ represents the negation of the one represented by $L_{i}\left(s_{i}\right)$. This negation is not representable by the label of a single state but rather by a union of $|A P|$ labels.

Example. Assume $A P=\left\{v_{0}, v_{1}, v_{2}\right\}$ and $\sigma=s_{0} \rightarrow s_{1}$ and $\widehat{L}\left(s_{0}\right)=\{\mathbf{f}, \mathbf{f}, \mathbf{f}\}$ the configuration associated with $s_{0}$ assigns false to each variable. The negation of this configuration represents a set of seven concrete configurations which are covered by three (abstract) configurations: $\{\{\mathbf{t}, \top, \top\},\{\mathbf{f}, \mathbf{t}, \top\},\{\mathbf{f}, \mathbf{f}, \mathbf{t}\}\}$.

To build the final AKS representing all sequences but spurious counterexample $\sigma$, one replaces in $A K S(\bar{\sigma})$ each state $\overline{s_{i}}$ by $k=\left|A P_{\bar{\sigma}}\right|$ states $\overline{s_{i}^{j}}$ with $j \in[0 . . k-$ 1] and assigns to each of them a label of $k$ variables $\left\{v_{0}, \ldots, v_{k-1}\right\}$ defined such that $: \widehat{L}\left(s_{i}^{j}\right)=\{\forall l \in[0 . . k-$ $\left.1], v_{l}=\neg L_{i}\left(s_{i}\right)\left[v_{l}\right], \forall l \in[j+1 . . k-1], v_{l}=\top\right\}$. Each state $s_{i}^{j}$ is connected to the same predecessor and successor states as state $\bar{s}_{i}$.

This final AKS presents a number of states in $\mathcal{O}(\mid$ $\sigma|\times| \mathcal{A P} \mid)$. However, removing, at each refinement step, the spurious counterexample only induces a low convergence. Moreover, in some cases, this strategy may not converge: suppose that all sequences of the form $a . b^{*} . c$ are spurious counterexamples (here $a$, $b$ and $c$ represent concrete state configurations). Assume, at a given refinement step $i$, a particular counterexample $\sigma_{i}=s_{0} \rightarrow s_{1} \rightarrow \ldots s_{n}$ with $L\left(s_{0}\right)=a, \forall k \in$ $[1, n-1], L\left(s_{k}\right)=b, L\left(s_{n}\right)=c$. Removing this counterexample does not prevent from a new spurious counterexample at step $i+1: \sigma_{i+1}=s_{0} \rightarrow s_{1} \rightarrow \ldots s_{n+1}$ with $L\left(s_{0}\right)=a, \forall k \in[1, n], L\left(s_{k}\right)=b, L\left(s_{n+1}\right)=c$. The strategy consisting of elimination spurious counterexample one by one diverges in this case. However, we cannot eliminate all the sequences of the form $a . b^{*} . c$ in a unique refinement step since we do not a priori know if at least one of these sequences is executable in the concrete model.

From these considerations, we are interested in removing sets of behaviors encompassing the spurious counterexample but still guaranteeing an over-approximation of the set of tree-organized behaviors of the concrete model. The strengthening of the abstraction $\widehat{M}_{i}$ with the addition of AKS of already verified local CTL properties eliminates sets of behaviors and guarantees the over-approximation (Property 2) but does not guarantee the elimination of the counterexample. We present in the following section a strategy to select sets of CTL properties eliminating the spurious counterexample.

\subsection{Ordering of properties}

We propose a heuristic to order the properties depending on the structure of each component. In order to do so, the variable dependency of the variables present in global property has to be analyzed. After this point, we refer to the variables present in the global property as primary variables.

We observed that the closer a variable is to the primary variable, the higher influence it has on it. Moreover, a global property often specifies the behavior at the interface of components. Typically, a global property ensures that a message sent is always acknowledged or the good target gets the message. This kind of behavior relates the input-output behaviors of components. We have decided to allocate an extra weight for interface variables whereas variables which do not interfere with a primary variable are weighted 0 . Here is how we proceed:

1. Build the structural dependency graph for all primary variables.

2. Compute the depth of all variables in all dependency graphs. Note that a variable may belong to more than one dependency graph, in that case we consider the minimum depth.

3. Give a weight to each variable (see Algorithm 1).

4. Compute the weight of properties for each component: sum of the property variables weight. 
The Algorithm 1 gives weight according to the variable distance to the primary variable with extra weight for interface variable and primary variable.

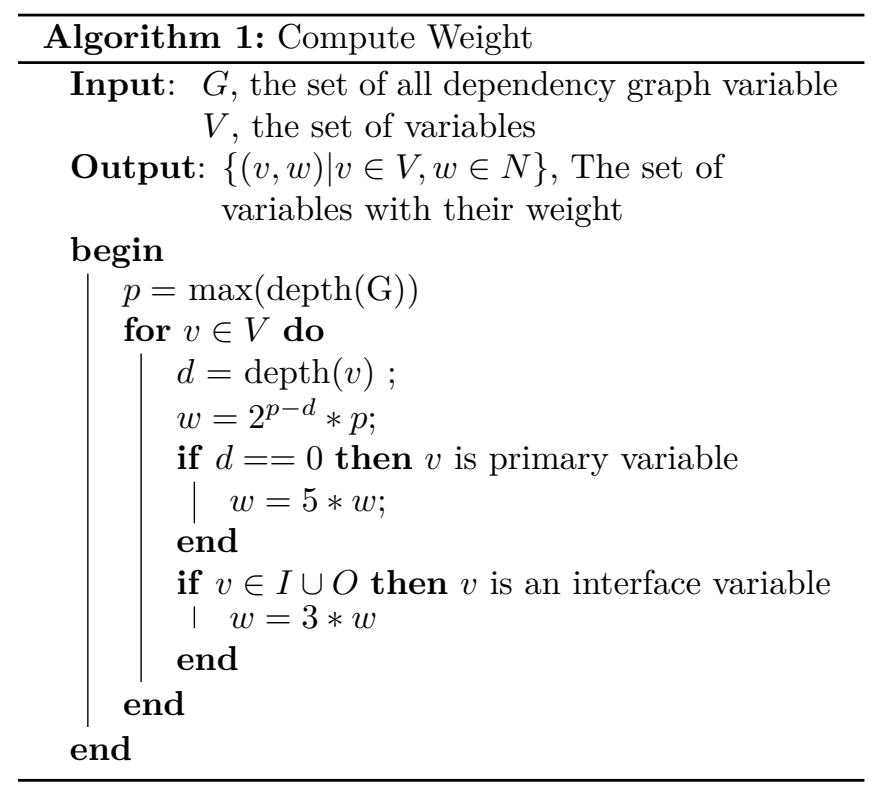

It is definitely not an exact pertinence calculation of properties but provides a good indicator of their possible impact on the global property. After this pre-processing phase, we have a list of properties ordered according to their pertinence with regards to the global property.

\subsection{Filtering properties}

The refinement step consists of adding new AKS of properties selected according to their pertinence. As we would like to ensure the elimination of the counterexample previously found, we filter out properties that do not have an impact on the counterexample $\sigma$ thus will not eliminate it. In order to reach this objective, a Abstract Kripke structure of the counterexample $\sigma, K(\sigma)$ is generated. $K(\sigma)$ is a succession of states corresponding to the counterexample path which dissatisfies the global property $\Phi$.

Definition 9. Let $\sigma$ be a counterexample of length $n$ in $\widehat{M}_{i}$ such that $\sigma=s_{0} \rightarrow s_{1} \rightarrow \ldots \rightarrow s_{n-1}$. The Kripke structure derived from $\sigma$ is 6-tuple $K\left(\sigma_{i}\right)=$ $\left(A P_{\sigma}, S_{\sigma}, S_{0 \sigma}, L_{\sigma}, R_{\sigma}, F_{\sigma}\right)$ such that:

- $A P_{\sigma}=\widehat{A P}_{i}:$ a finite set of atomic propositions which corresponds to the variables in the abstract model

- $S_{\sigma}=\left\{s_{i} \mid s_{i} \in \sigma\right\} \cup\left\{s_{T}\right\}$

- $S_{0 \sigma}=\left\{s_{0}\right\}$

- $L_{\sigma}=\widehat{L}_{i} \cup L\left(s_{T}\right)=\left\{\top, \forall p \in A P_{\sigma}\right\}$

- $R_{\sigma}=\left\{\left(s_{k}, s_{k+1}\right) \mid\left(s_{k} \rightarrow s_{k+1}\right) \in \sigma\right\} \cup\left\{\left(s_{n-1}, s_{T}\right)\right\}$

- $F_{\sigma}=\emptyset$
All the properties available for refinement are then model-checked on $K(\sigma)$. If the property holds then the property will not eliminate the counterexample. Hence this property is not a good candidate for refinement. Therefore the highest weighted property not satisfied in $K(\sigma)$ is chosen to be integrated in the next refinement step. This process is iterated for each refinement step.

Property 3. Counterexample eviction

1. If $K(\sigma) \vDash \varphi \Rightarrow A K S(\varphi)$ will not eliminate $\sigma$.

2. If $K(\sigma) \not \models \varphi \Rightarrow A K S(\varphi)$ will eliminate $\sigma$.

Proof. 1. By construction, $A K S(\varphi)$ simulates all models that verify $\varphi$. Thus the tree described by $K(\sigma)$ is simulated by $A K S(\varphi)$, it implies that $\sigma$ is still a possible path in $A K S(\varphi)$.

2. $K(\sigma)$, where $\varphi$ does not hold, is not simulated by $A K S(\varphi)$, thus $\sigma$ is not a possible path in $A K S(\varphi)$ otherwise $A K S(\varphi) \not \models \varphi$ that is not feasible due to AKS definition and the composition with $M_{i}$ with $A K S(\varphi)$ will eliminate $\sigma$.

The proposed approach ensures that the refinement excludes the counterexample and respects the Definition 6 . We will show in our experiments that first, the time needed to build an AKS is negligible and secondly the refinement converges rapidly.

\section{EXPERIMENTAL RESULTS}

We have conducted preliminary experiments to test and compare the performance of our strategy with existing techniques available in VIS. There are several abstractionrefinement techniques implemented in VIS accessible via approximate_model_check, iterative_model_check, check_invariant and incremental_ctl_verification commands. However, among the available techniques, incremental_ctl_verification is the only one that supports CTL formulas and fairness constraints which are necessary in our test platforms. It is an automatic abstraction refinement algorithm which generates an initial conservative abstraction principally by reducing the size of the latches by a constant factor. If the initial abstraction is not conclusive, a goal set will then be computed in order to guide the refinement process [20] [21].

We have executed and compared the execution time and the number of refinement iterations for two examples: VCI-PI platform consisting of Virtual Component Interface (VCI), a PI-Bus and VCI-PI protocol converter and a simplified version of a CAN bus platform consisting of 3 nodes on a CAN bus. Table 1 gives the size and the statistics concerning the VCI-PI platform and CAN bus platform verified. All the values are obtained using the compute_reach command with option $-v 1$ in VIS 
except the number of BDD variables, computed using the print_bdd_stats command. The experiments have been executed on a PC with an AMD Athlon dual-core processor $4450 \mathrm{e}$ and $1.8 \mathrm{~GB}$ of RAM memory.

\begin{tabular}{|c|c|c|c|c|}
\hline $\begin{array}{l}\text { Experim. } \\
\text { Platform }\end{array}$ & $\begin{array}{c}\text { Global } \\
\text { Property }\end{array}$ & $\begin{array}{c}\text { Verification } \\
\text { Technique }\end{array}$ & $\begin{array}{c}\text { Refine. } \\
\text { Iter. }\end{array}$ & $\begin{array}{c}\text { Verif. } \\
\text { Time (s) }\end{array}$ \\
\hline \multirow{6}{*}{$\begin{array}{c}\text { VCI-PI: } \\
1 \text { Master } \\
- \\
1 \text { Slave }\end{array}$} & \multirow{3}{*}{$\phi_{1}$} & Prop. Select. & 1 & 2.2 \\
\hline & & Incremental & 0 & 6.3 \\
\hline & & Standard MC & - & 6.06 \\
\hline & \multirow{3}{*}{$\phi_{2}$} & Prop. Select. & 0 & 1.0 \\
\hline & & Incremental & 562 & 200.9 \\
\hline & & Standard MC & - & 6.13 \\
\hline \multirow{6}{*}{$\begin{array}{c}\text { VCI-PI: } \\
2 \text { Masters } \\
- \\
1 \text { Slave }\end{array}$} & \multirow{3}{*}{$\phi_{1}$} & Prop. Select. & 1 & 2.0 \\
\hline & & Incremental & 0 & 20.4 \\
\hline & & Standard MC & - & 37.9 \\
\hline & \multirow{3}{*}{$\phi_{2}$} & Prop. Select. & 0 & 1.0 \\
\hline & & Incremental & 74 & 786.3 \\
\hline & & Standard MC & - & 39.4 \\
\hline \multirow{6}{*}{$\begin{array}{c}\text { VCI-PI: } \\
4 \text { Masters } \\
- \\
1 \text { Slave }\end{array}$} & \multirow{3}{*}{$\phi_{1}$} & Prop. Select. & 1 & 2.1 \\
\hline & & Incremental & 0 & 261.6 \\
\hline & & Standard MC & - & $>1$ day \\
\hline & \multirow{3}{*}{$\phi_{2}$} & Prop. Select. & 0 & 1.0 \\
\hline & & Incremental & 0 & 263.5 \\
\hline & & Standard MC & - & $>1$ day \\
\hline \multirow{6}{*}{$\begin{array}{c}\text { VCI-PI: } \\
4 \text { Masters } \\
- \\
2 \text { Slaves }\end{array}$} & \multirow{3}{*}{$\phi_{1}$} & Prop. Select. & 1 & 2.2 \\
\hline & & Incremental & $\mathrm{N} / \mathrm{A}$ & $>1$ day \\
\hline & & Standard MC & - & $>1$ day \\
\hline & \multirow{3}{*}{$\phi_{2}$} & Prop. Select. & 0 & 1.1 \\
\hline & & Incremental & $\mathrm{N} / \mathrm{A}$ & $>1$ day \\
\hline & & Standard MC & - & $>1$ day \\
\hline \multirow{6}{*}{$\begin{array}{l}\text { CAN } \\
\text { Bus }\end{array}$} & \multirow{3}{*}{$\phi_{3}$} & Prop. Select. & 0 & 1.02 \\
\hline & & Incremental & $\mathrm{N} / \mathrm{A}$ & $>1$ day \\
\hline & & Standard MC & - & 2645.4 \\
\hline & \multirow{3}{*}{$\phi_{4}$} & Prop. Select. & 0 & 1.01 \\
\hline & & Incremental & $\mathrm{N} / \mathrm{A}$ & $>1$ day \\
\hline & & Standard MC & - & 1678.1 \\
\hline
\end{tabular}

Table 2. Verification Results

In Table 2, we compare the execution time and the number of refinement between our technique (Prop. Select.), incremental_ctl_verification (Incremental) and the standard model checking (Standard MC) computed using the model_check command in VIS (Note: Dynamic variable ordering has been enabled with sift method). For the VCI-PI platform, the global property $\phi_{1}$ is the type $A F((p=1) * A F(q=1))$ and $\phi_{2}$ is actually a stronger version of the same formula with $A G(A F((p=1) * A F(q=$ $1)))$. We have a total of 26 verified components properties to be selected in the VCI-PI platform. In comparison to $\phi_{2}$, we can see that, a better set of properties available will result in a better abstraction and less refinement iterations.

In the case of the CAN bus platform, the global prop- erty $\phi_{3}$ is the type $A G\left(\left(\left(p^{\prime}=1\right) *\left(q^{\prime}=1\right) * A F\left(r_{1}=\right.\right.\right.$ $\left.1)) \rightarrow A F\left(\left(s_{1}=1\right) * A F\left(t_{1}=1\right)\right)\right)$ and $\phi_{4}=A G\left(\left(\left(p^{\prime}=\right.\right.\right.$ $\left.\left.1) *\left(q^{\prime}=1\right) * A G\left(r_{2}=0\right)\right) \rightarrow A G\left(\left(s_{2}=0\right) *\left(t_{2}=0\right)\right)\right)$. We have at our disposal 103 verified component properties and after the selection process for the initial abstraction, 3 selected component properties were sufficient to verify both global properties without refinement.

Globally, we can see that our technique, for these examples, systematically computes faster than the other two methods and interestingly in the case where the size of the platform increases by adding more connected components, in contrary to the other two methods, our computation time remains stable. This is mainly due to the fact that for small number of properties, our abstraction is generated almost instantly and as only pertinent properties are selected, not many refinement iterations are required in order to complete the verification process. It is also important to note that the properties tested are simple and we have in our property selection list the local properties required to satisfy the global property.

\section{CONCLUSION AND FUTURE WORKS}

We have presented a new strategy in the abstraction generation and refinement which is well adapted for compositional embedded systems. This verification technique is compatible and suits well in the natural development process of complex systems. Our preliminary experimental results show an interesting performance in terms of duration of abstraction generation and the number of refinement iteration. Furthermore, this technique enables us to overcome repetitive counterexamples due to the presence of cycles in the system's graph.

Nevertheless, in order to function well, this refinement technique requires a well constituted specification of every components of the concrete model. Furthermore, it may be possible that none of the properties available is capable of eliminating the counterexample which is probably due to an incomplete specification or a counterexample that should be eliminated by the product of local properties. In this case, other refinement techniques such as the refinement by eliminating the counterexample only, or the identification of a good set of local properties to be integrated simultaneously, should be considered. We are currently investigating other complementary techniques to overcome these particular cases. The work of Kroening [22], for example, could also help us in improving the specification of the model: at the component level, or for groups of components.

\section{REFERENCES}

[1] The VIS Group, "VIS: A system for Verification and Synthesis," Springer LNCS, vol. 1102, pp. 428-432, 1996. 


\begin{tabular}{|c|c|c|c|c|c|c|}
\hline \multicolumn{3}{|c|}{$\begin{array}{l}\text { Experiment } \\
\text { Platform }\end{array}$} & $\begin{array}{c}\text { Number of } \\
\text { BDD Variables }\end{array}$ & $\begin{array}{l}B D D \\
\text { Size }\end{array}$ & $\begin{array}{c}\text { Number of } \\
\text { Reachable States }\end{array}$ & $\begin{array}{l}\text { Analysis } \\
\text { Time (s) }\end{array}$ \\
\hline \multirow{12}{*}{ VCI-PI } & \multirow{4}{*}{$\begin{array}{l}\text { Concrete } \\
\text { Model }\end{array}$} & 1 Master-1 Slave & 304 & 7207 & $4.711 \mathrm{e}+3$ & 6.36 \\
\hline & & 2 Masters-1 Slave & 445 & 24406 & $7.71723 \mathrm{e}+06$ & 35.2 \\
\hline & & 4 Masters-1 Slave & 721 & 84118 & $3.17332 \mathrm{e}+12$ & 2818.3 \\
\hline & & 4 Masters-2 Slaves & 895 & 238990 & $5.708 \mathrm{e}+15$ & $68882.3^{1}$ \\
\hline & Final & 1 Master-1 Slave & 197 & 76 & $5.03316 \mathrm{e}+07$ & 0.01 \\
\hline & Abstract & 2 Masters-1 Slave & 301 & 99 & $4.12317 \mathrm{e}+11$ & 0.02 \\
\hline & Model & 4 Masters-1 Slave & 501 & 147 & $3.45876 \mathrm{e}+18$ & 0.03 \\
\hline & for $\phi_{1}$ & 4 Masters-2 Slaves & 589 & 167 & $7.08355 \mathrm{e}+21$ & 0.04 \\
\hline & Final & 1 Master-1 Slave & 194 & 50 & $2.62144 \mathrm{e}+07$ & 0 \\
\hline & Abstract & 2 Masters-1 Slave & 298 & 73 & $2.14748 \mathrm{e}+11$ & 0.01 \\
\hline & Model & 4 Masters-1 Slave & 498 & 121 & $1.80144 \mathrm{e}+18$ & 0.02 \\
\hline & for $\phi_{2}$ & 4 Masters-2 Slaves & 586 & 141 & $3.68935 \mathrm{e}+21$ & 0.02 \\
\hline \multirow{3}{*}{ CAN Bus } & \multicolumn{2}{|c|}{ Concrete Model } & 822 & 161730 & $3.7354 \mathrm{e}+07$ & 300.12 \\
\hline & \multicolumn{2}{|c|}{ Final Abstract Model for $\phi_{3}$} & 425 & 187 & $1.66005 \mathrm{e}+12$ & 0.03 \\
\hline & \multicolumn{2}{|c|}{ Final Abstract Model for $\phi_{4}$} & 425 & 187 & $1.66005 \mathrm{e}+12$ & 0.04 \\
\hline
\end{tabular}

Table 1. Statistics on the VCI-PI and CAN Bus platform

[2] S. Graf and H. Saïdi, "Construction of Abstract State Graphs with PVS," in Computer Aided Verification (CAV'97). 1997, vol. 1254, LNCS, Springer.

[3] E. M. Clarke, O. Grumberg, S. Jha, Y. Lu, and H. Veith, "Counterexample-guided Abstraction Refinement," in CAV'00, Chicago, IL, 2000, LNCS.

[4] T. Ball, B. Cook, V. Levin, and S. K. Rajamani, "SLAM and Static Driver Verifier: Technology Transfer of Formal Methods inside Microsoft," in 4th Int. Conf. on Integrated Formal Methods. 2004, vol. 2999, pp. 1-20, Springer.

[5] D. Beyer, T. A. Henzinger, R. Jhala, and R. Majumdar, "The Software Model Checker Blast: Applications to software engineering," in Int. Journal on Software Tools for Technology Transfer, 2007, vol. 9 (5-6), pp. 505-525.

[6] H. Jain, D. Kroening, N. Sharygina, and E. Clarke, "VCEGAR: Verilog CounterExample Guided Abstraction Refinement," in TACAS'0\%, 2007.

[7] N. Sharygina, S. Tonetta, and A. Tsitovich, "An abstraction refinement approach combining precise and approximated techniques," International Journal on Software Tools for Technology Transfer, vol. 14, pp. 1-14, 2012.

[8] O. Grumberg and D. E. Long, "Model Checking and Modular Verification," in Int. Conference on Concurency Theory. 1991, vol. 527, pp. 250-263, Springer-Verlag.

[9] T. A. Henzinger, S. Qadeer, and S. K. Rajamani, "You Assume, We Guarantee: Methodology and Case Studies," in CAV'98, Canada, 1998, vol. 1427, pp. 440-451, Springer-Verlag.

[10] F. Xie and J.C. Browne, "Verified Systems by Composition from Verified Components," in ESEC/FSE 2003: 11th ACM SIGSOFT Symposium on Foundations of Software Eng. Conf., Finland, 2003, pp. 227-286, ACM Press.

[11] J. Li, X. Sun, F. Xie, and X. Song, "Component-Based Abstraction Refinement," in 10th Int. Conf. on Software Reuse (ICSR), China, 2008, pp. 39-51, Springer-Verlag.

[12] H. Peng, Y. Mokhtari, and S. Tahar, "Environment Synthesis for Compositional Model Checking," in ICCD'02: 20th Int. Conference on Computer Design, Freiburg, Germany, 2002, pp. 70-75, IEEE Computer Society.
[13] M. Schickel, V. Nimbler, M. Braun, and H. Eveking, "On Consistency and Completeness of Property-Sets: Exploiting the Property-Based Design Process," in FDL'06: Forum on specification and Design Languages, 2006.

[14] M. D. Nguyen, M. Wedler, D. Stoffel, and W. Kunz, "Formal Hardware/Software Co-Verification by Interval Property Checking with Abstraction," in Design Automation Conference (DAC'11), San Diego, USA, 2011.

[15] C. Braunstein and E. Encrenaz, "Using CTL Formulae as Component Abstraction in a Design Verification Flow," ACSD IEEE Computer Society Press, pp. 80-89, 2007.

[16] A. Bara, "Abstraction de Composant pour la Vérification par Model-Checking," Mémoire de Diplôme Universitaire OMP - LIP6-SOC, 2008.

[17] E. M. Clarke, O. Grumberg, and D.E. Long, "Model checking and abstraction," ACM Transactions on Programming Languages and Systems, vol. 16, no. 5, pp. 1512-1542, 1994.

[18] N. Belnap, "A useful four-valued logic," Modern Uses of Multiple-Valued Logic, pp. 8-37, 1977.

[19] C. Braunstein, "Conception Incrémentale, Vérification de Composants Matériels et Méthode d'abstraction pour la Vérification de Systèmes Intégrés sur Puce", Ph.D. thesis, Université Pierre et Marie Curie, LIP6-SOC, 2007.

[20] S. Pardo and G. Hachtel, "Incremental CTL Model Checking Using BDD Subsetting," in DAC '98: 35th Design Automation Conference, 1998, pp. 457-462.

[21] S. Pardo and G. Hachtel, "Automatic Abstraction Technique for Propositional mu-Calculus Model Checking," in $C A V^{\prime} 97$. 1997, vol. 1254, pp. 12-23, Springer-Verlag.

[22] M. Purandare, T. Wahl, and D. Kroening, "Strengthening properties using abstraction refinement," in Proceedings of DATE 2009. 2009, pp. 1692-1697, ACM.

\footnotetext{
${ }^{1}$ Computed on a calculation server: $2 \mathrm{x}$ Xeon X5650, 72Go RAM
} 